\title{
Comparative Analysis of Optical Ring Resonators with Cross- and Direct-Coupled Configurations
}

\author{
Faramarz E. Seraji ${ }^{1,2, ~ *, ~ F a t e m e h ~ A s g a h r i ~}{ }^{3}$ \\ ${ }^{1}$ Optical Communication Group, Iran Telecom Research Center, Tehran, Iran \\ ${ }^{2}$ Electrical Eng. Dept., Ghiasodin Jamshid Kashani Institute of Higher Edu., Abyek, Qazvin, Iran \\ ${ }^{3}$ Physics Group, Payam Noor University, Shahr Rey, Iran
}

\begin{abstract}
This paper presents a proposed alternate configuration for optical ring resonator (ORR) having a resonance loop made of single-mode optical fiber. Under steady state condition, the responses of the proposed ORR are formulated by considering the output and loop intensities for various conditions of transmission coefficient, power coupling coefficient, and compared the results with the previous reported ORR configuration. The analyses have shown that the main difference between two ORRs is due to the values of power coupling coefficients of the couplers used in their configurations. Another major point, that is to be considered in design of ORR for a maximum output power is that the transmission coefficient and power coupling coefficient in the proposed configuration are not required to be equal, as the case of the previously reported configuration. The analytical results given in this article can be useful for selection of ORR configuration for particular applications, specially in case of nonlinear use of ORR configuration.
\end{abstract}

Keywords Comparative analysis, Optical ring resonator, Alternate configurations

\section{Introduction}

With increasing demands in optical communication and sensing systems, optical ring resonators (ORR) with different structures[1-6], have been analyzed and proposed for several applications such as polarization sensing[7], biosensing[8], optical filters[9-14], optical fiber dispersion compensation devices[15,16], optical integration/ differentiation and optical triggering[2]. optical bistability[17,18], add/ drop multiplexer[19], optical switching[20-23], and various other applications[24-30]. In early work, steady state[2,31] and dynamic responses of ORR built on fiber were analyzed $[32,33]$ for applications in polarization sensing[7], FM deviation measurement of a laser diode[33], optical triggering, optical integration/differentiation and fiber dispersion compensation[2], and rotation sensing[31]. Recently, dynamic resonance characteristic of fiber ring resonator has been analyzed for gyro system[34].

A basic structure of an ORR consists of a $2 \times 2$ directional coupler where one of the input port is connected to one of the output port, making a closed loop to configure a ring resonator. To operate an ORR at resonance, the loop length could be of the order of few micrometers[10] to tens of meters[32].

Recently, a full steady-state analysis of the ORR is

* Corresponding author:

feseraji@itrc.ac.ir (Faramarz E. Seraji)

Published online at http://journal.sapub.org/optics

Copyright (C) 2012 Scientific \& Academic Publishing. All Rights Reserved reported where characteristic parameters such as coupling coefficient of the coupler and transmission parameters of the loop fiber affecting the response are investigated[35]. In another report, the dynamic behavior of the ORR, by considering the influences of light-source parameters on the response, is presented[36].

An ORR may be realized mainly by two types of configurations: Cross-coupled and direct-coupled. To the best of our knowledge, in the reported analyses of the ORR in the literatures, the importance and difference of these configurations have not been addressed with application point of views. With respect to the type of high power applications of ORR, variety of configuration may play an important role[37-39].

In this paper, we analyze the two ORR configurations and compare their characteristic intensities at the output and in the resonance loop[35]. It is shown that in one configuration the, the resonance loop intensity is about twice the other one.

\section{ORR Configurations and Response Formulation}

\subsection{Intensities of Output and Resonance Loop}

The two ORR configurations, which are considered for analysis, are shown in Fig. 1. In Fig. 1(a), a previously reported configuration[35] is shown in which the resonance loop is made by connecting two cross-coupled ports (2) and (3), whereas the resonance loop of our suggested ORR con- 
figuration in Fig. 1(b) is made by connecting two direct-coupled ports (2) and (4).

For cross-coupled ORR (CCORR) in Fig. 1(a), let us assume that the loop and the coupler are ideal with no polarization coupling and the birefringence in the loop fiber is zero. In Fig. 1(a), if we denote $E_{\text {in }}, E_{\mathrm{r} 2}, E_{\mathrm{r} 1}$ and $E_{0}$ the electric fields of light waves at ports (1), (2), (3), and (4), respectively, the complex field amplitudes at ports (3) and (4), under steady state operation, can be expressed as[1,35].

$$
\begin{aligned}
& E_{r 1}=\sqrt{1-\gamma_{o}}\left(\sqrt{1-\kappa} E_{i n}-j \sqrt{\kappa} E_{r 2}\right) \\
& E_{o}=\sqrt{1-\gamma_{o}}\left(-j \sqrt{\kappa} E_{i n}+\sqrt{1-\kappa} E_{r 2}\right)
\end{aligned}
$$

Where $\kappa$ is the power coupling coefficient of the coupler and $\gamma_{0}$ is its fractional intensity loss.

For an ideal condition and based on conservation of energy[35,40], the electric fields $E_{\mathrm{r} 2}$ and $E_{\mathrm{r} 1}$ are related by:

$$
\begin{gathered}
\left|E_{\mathrm{r} 2}\right|^{2}=\alpha\left|E_{\mathrm{r} 1}\right|^{2} \\
E_{\mathrm{r} 2}=\sqrt{\alpha} E_{\mathrm{r} 1} \exp \left(-j \omega_{\mathrm{c}} \tau\right)
\end{gathered}
$$

where $\tau$ is the loop delay time, $\omega_{c}$ is the optical angular frequency, and $\alpha$ is the power transmission coefficient of the loop fiber expressed as $\log \alpha=-\alpha_{0} L$, where $\alpha_{0}$ is the attenuation coefficient (in $\mathrm{dB} / \mathrm{m}$ ) of the loop fiber, $L$ is the length of the loop (in m).
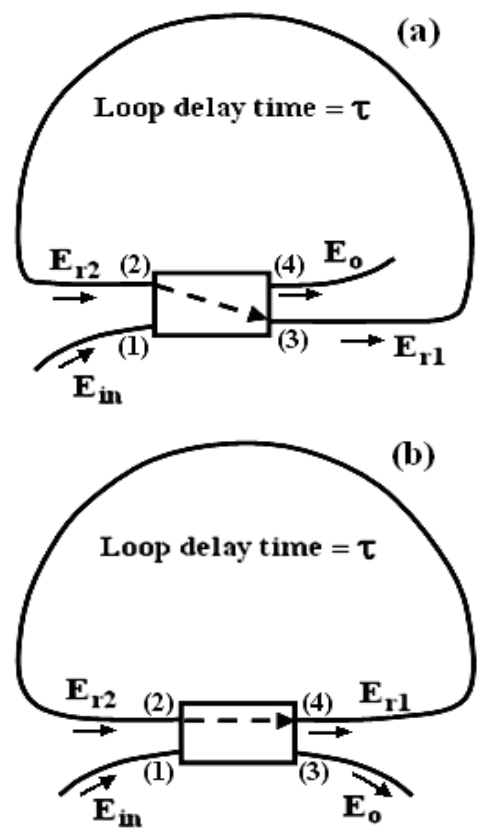

Figure 1. Schematic diagrams of optical ring resonators (a) cross-coupled and (b) direct-coupled configurations

From Eqs. (1), (2), and (4) we can obtain the field at port $2[35]:$

$$
E_{\mathrm{r} 2}=\left\{\frac{\sqrt{\alpha} \sqrt{1-\gamma_{0}} \sqrt{1-\kappa_{0}}}{\exp \left(j \omega_{\mathrm{c}} \tau\right)+j \sqrt{\alpha \kappa\left(1-\gamma_{0}\right)}}\right\} E_{\mathrm{in}}
$$

Substituting Eqs. (1), (4), and (5) in Eq. (2), $E_{0}$ results in:

$$
E_{0}=\sqrt{1-\gamma_{\mathrm{o}}}\left\{\frac{\sqrt{\alpha} \sqrt{1-\gamma_{0}}-j \sqrt{\kappa} \exp \left(j \omega_{\mathrm{c}} \tau\right)}{\exp \left(j \omega_{\mathrm{c}} \tau\right)+j \sqrt{\alpha \kappa\left(1-\gamma_{0}\right)}}\right\} E_{\mathrm{in}}
$$

\subsection{Intensities of Output and Resonance Loop}

By some algebraic manipulations of Eqs. (5) and (6), the output intensity $I_{\text {out }}$ at port (4) and the loop intensity $I_{\text {loop }}$ at port (2) both normalized to input intensity can be shown, respectively, as:

$$
\begin{gathered}
I_{\text {out }}=\left|E_{0} / E_{\text {in }}\right|^{2} \\
I_{\text {out }}=\left(1-\gamma_{0}\right)\left\{1-\frac{(1-\kappa)\left[1-\alpha\left(1-\gamma_{0}\right)\right]}{1+\alpha \kappa\left(1-\gamma_{0}\right)+2 \sqrt{\alpha \kappa\left(1-\gamma_{0}\right)} \sin \left(\omega_{\mathrm{c}} \tau\right)}\right\} \\
I_{\text {loop }}=\left|E_{\mathrm{r} 2} / E_{\text {in }}\right|^{2} \\
I_{\text {loop }}=\frac{\alpha\left(1-\gamma_{0}\right)(1-\kappa)}{1+\alpha \kappa\left(1-\gamma_{0}\right)+2 \sqrt{\alpha \kappa\left(1-\gamma_{0}\right)} \sin \left(\omega_{\mathrm{c}} \tau\right)}
\end{gathered}
$$

\subsection{Condition for Resonance}

The condition for resonance in the ORR is obtained by finding the value of $\omega_{c}$ at which the loop intensity $I_{\text {loop }}$ builds up to a maximum value and the output intensity $I_{\text {out }}$ reduces to a minimum value. By inspection of Eq. (8), the condition for resonance is found to be $\omega_{\mathrm{c}} \tau=2 q \pi-\pi / 2$, where $q$ is an integer. Equation (8) shows that at resonance, the value of the output intensity $I_{\text {out }}$ becomes a minimum. The additional condition required to make $I_{\text {out }}$ to become zero at resonance can be shown as:

$$
\kappa=\alpha\left(1-\gamma_{0}\right)
$$

If the coupler is lossless $\left(\gamma_{0}=0\right)$, we have $\kappa=\alpha$. Figure 2 shows the theoretical output and loop intensities of the CCORR as a function of $\omega_{c} \tau$ for different values of $\kappa(=\alpha)$.

We note that for all values of $\kappa$, the output response dips to zero, and loop intensity peaks to a maximum value, indicating the resonance condition. As the value of $\kappa(=\alpha)$ decreases, the sharpness of the resonance response decreases as well.

When $\kappa=\alpha\left(1-\gamma_{0}\right)$, it can be shown that at resonance when $\omega_{\mathrm{c}} \tau=2 q \pi-\pi / 2$, the maximum developed loop intensity $I_{\text {loop, max }}$ is:

$$
I_{\text {loop, } \max }=\frac{\kappa}{1-\kappa}
$$

The maximum value of the normalized loop intensity in this case is 19 for $\kappa=\alpha=0.95$. A reduction of 0.2 in the value of $\kappa(=\alpha)$ from 0.95 to 0.75 causes the maximum loop intensity to decrease from 19 to 3 . We note that in Fig. 2(b) the resonance response is not sharp when the value of $\kappa(=\alpha)$ is small.

\subsection{Response of CCORR at Resonance}

From Eq. (8), at $\omega_{\mathrm{c}} \tau=2 q \pi-\pi / 2$ and $\gamma_{0}=0$, the normalized output intensity can be shown by:

$$
\left.I_{\text {out, }, \text { CORR }}\right|_{\omega_{\mathrm{c}} \tau=-\pi / 2}=\frac{(\sqrt{\kappa}-\sqrt{\alpha})^{2}}{(1-\sqrt{\alpha \kappa})^{2}}
$$



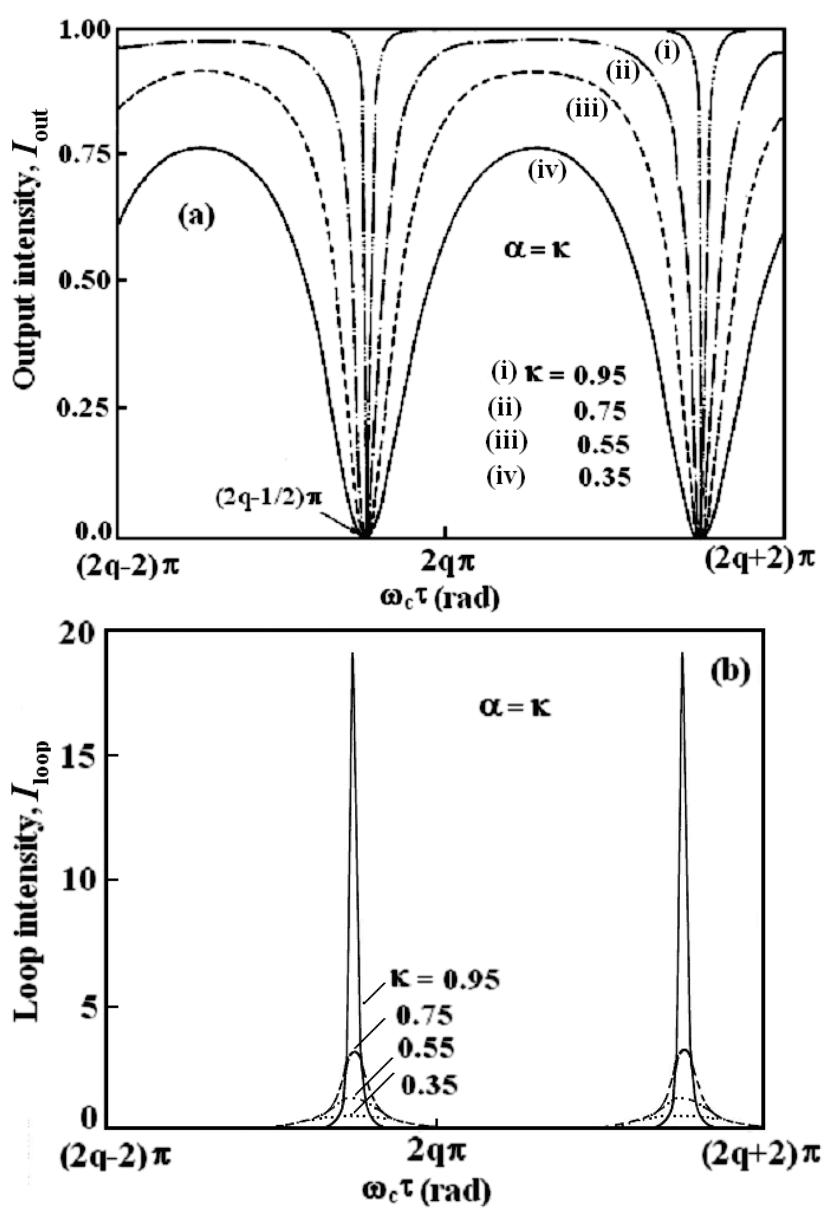

Figure 2. Normalized (a) output intensity and (b) loop intensity of CCORR with respect to $\omega_{\mathrm{c}} \tau$ when $\gamma_{0}=0$ and $\kappa=\alpha=0.95,0.75,0.55$, and 0.35

From Eq. (10), $I_{\text {loop }}$ can be derived as:

$$
\left.I_{\text {loop, CCORR }}\right|_{\omega_{\mathrm{c}} \tau=-\pi / 2}=\frac{\alpha(1-\kappa)}{(1-\sqrt{\alpha \kappa})^{2}}
$$

Equation (13) gives the minimum value of $I_{\text {out }}$ at $\omega_{\mathrm{c}} \tau=2 q \pi-\pi / 2$ as a function of $\kappa$ and $\alpha$. The normalized output intensity is equal to $\kappa$ for $\alpha=0$, is zero when $\kappa=\alpha$, and is unity at $\kappa=\alpha$. According to Eq. (14), under the resonance condition, if $\kappa=\alpha$, the theoretical value of $I_{\text {loop }}$ tends to be large when $\kappa$ tends to unity. But when $\kappa=1$, the power coupling in the coupler of the CCORR will be 100 percent, resulting in a zero loop intensity.

\section{Suggested Configuration of ORR}

In this section, we analyze our suggested configuration of an ORR, as presented in Fig. 1(b), which is hereafter termed as DCORR. Principally, this configuration has a response similar to that of the CCORR discussed so far. The difference between the configurations of Fig. 1(b) and of Fig 1(a) is that in former port (4) is used for feedback loop instead of port (3) as in the latter case.

Under steady state operation, as in the previous section, the complex electric field at ports (3) and (4) of Fig. 1(b) can respectively be written as:

$$
\begin{gathered}
E_{\mathrm{r} 1}=\sqrt{1-\gamma_{0}}\left(-j \sqrt{\kappa} E_{\mathrm{in}}+\sqrt{1-\kappa} E_{\mathrm{r} 2}\right) \\
E_{0}=\sqrt{1-\gamma_{0}}\left(\sqrt{1-\kappa} E_{\mathrm{in}}-j \sqrt{\kappa} E_{\mathrm{r} 2}\right) \\
\left|E_{\mathrm{r} 2}\right|^{2}=\alpha\left|E_{\mathrm{r} 1}\right|^{2}
\end{gathered}
$$

where all the parameters have their usual meaning. In a similar procedure, the normalized loop and output intensities can be shown as:

$$
\begin{aligned}
& I_{\text {loop }}=\left|E_{r 2} / E_{\text {in }}\right|^{2} \\
& =\frac{\alpha \kappa\left(1-\gamma_{0}\right)}{1+\alpha\left(1-\gamma_{0}\right)(1-\kappa)-2 \sqrt{\alpha\left(1-\gamma_{0}\right)(1-\kappa)} \cos \left(\omega_{\mathrm{c}} \tau\right)} \\
& I_{\text {out }}=\left|E_{0} / E_{\text {in }}\right|^{2} \\
& =\left(1-\gamma_{0}\right)\left\{\frac{(1-\kappa)+\alpha\left(1-\gamma_{0}\right)-2 \sqrt{\alpha\left(1-\gamma_{0}\right)(1-\kappa)} \cos \left(\omega_{\mathrm{c}} \tau\right)}{1+\alpha\left(1-\gamma_{0}\right)(1-\kappa)-2 \sqrt{\alpha\left(1-\gamma_{0}\right)(1-\kappa)} \cos \left(\omega_{\mathrm{c}} \tau\right)}\right\}
\end{aligned}
$$

\subsection{Response of DCORR at Resonance}

The normalized loop intensity at resonance the condition $\omega_{\mathrm{c}} \tau=2 q \pi, q=0,1,2, \ldots$ and $\gamma_{0}=0$ from Eq. (18) can be shown as:

$$
\begin{aligned}
\left.I_{\text {loop, DCORR }}\right|_{\omega_{\mathrm{c}} \tau=0} & =\frac{\alpha \kappa}{(1-\sqrt{\alpha(1-\kappa)})^{2}} \\
\left.I_{\text {out, DCORR }}\right|_{\omega_{\mathrm{c}} \tau=0} & =\frac{(\sqrt{\alpha}-\sqrt{(1-\kappa)})^{2}}{(1-\sqrt{\alpha(1-\kappa)})^{2}}
\end{aligned}
$$

In general, Eq. (21) gives the minimum value of the out put intensity as function of $\kappa$ and $\alpha$. The additional condition for the output intensity $I_{\text {out }}$ to become zero at resonance is given by:

$$
\kappa=1-\alpha\left(1-\gamma_{0}\right)
$$

For $\gamma_{0}=0$ will be:

$$
\kappa=1-\alpha
$$

Figure 3 shows the normalized output intensity $I_{\text {out }}$ of the DCORR as a function of $\omega_{\mathrm{c}} \tau$ and Fig. 4 illustrates the normalized loop intensity $I_{\text {loop }}$, assuming $\alpha=1-\kappa$ and $\gamma_{0}=0$.

\section{Comparison of CCORR and DCORR}

If we compare Fig. 1 with Fig. 2, the responses of the CCORR and the DCORR indicate that the behavior is the same in both the cases except that the resonance in the former occurs at $\omega_{\mathrm{c}} \tau=2 q \pi-\pi / 2, q=0,1,2, \ldots$ whereas in the latter it occurs at $\omega_{\mathrm{c}} \tau=2 q \pi, q=0,1,2, \ldots$. The major difference between two configurations lies in the value of $\kappa$, where in the DCORR, when value of $\kappa$ is lower, the finesse of the resonator will be higher, while in case of the CCORR is reverse. Moreover, we note that at resonance conditions, at whatsoever value it maybe, the loop intensity in CCORR is nearly twice the value in the DCORR.

For practical reasons, the CCORR configuration in Fig. 1 (a) is usually employed, since such a configuration can be 
fabricated by using a single length of an optical fiber without a need of any splice joint. To obtain high finesse or to make $\alpha$ as large as possible, it is desirable to avoid a splice joint in the loop fiber. To fabricate the configuration of DCORR in Fig. 1(b), two separate lengths of optical fibers would be needed to realize the coupler and a splice joint would be required to make the loop.
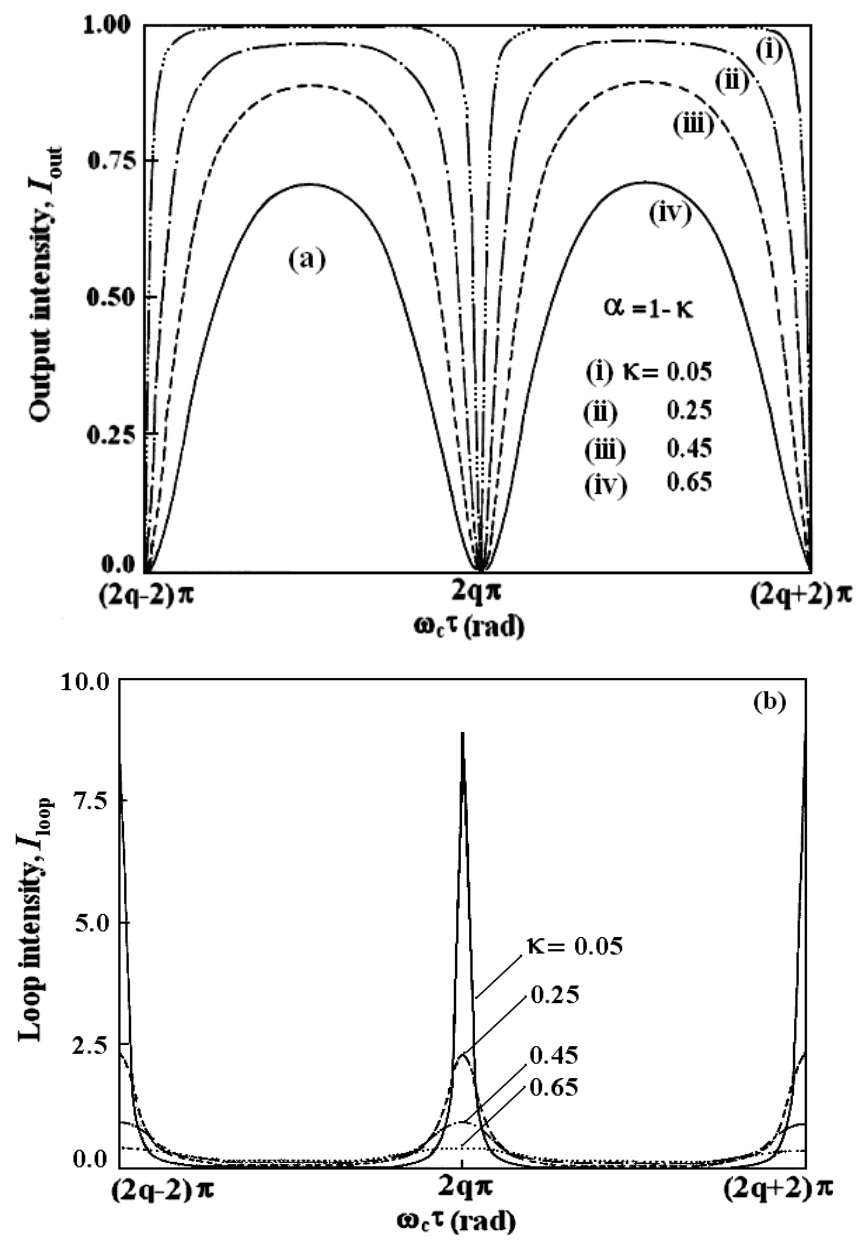

Figure 3. Normalized (a) output intensity and (b) loop intensity of DCORR with respect to $\omega_{\mathrm{c}} \tau$ when $\gamma_{0}=0$ and $\alpha=1-\kappa=0.05,0.25,0.45$, and 0.65

\section{Conclusions}

This paper suggested a configuration for optical ring resonator having a resonance loop made of single-mode optical fiber. Under steady state condition, the responses of the proposed ORR are formulated by considering the output and loop intensities for various conditions of transmission coefficient $\alpha$, power coupling coefficient $\kappa$, and compared the results with the previously reported ORR configuration.

The analyses have shown that the characteristic behaviors of both the configurations are the same. The main difference between two ORRs is due to the value of power coupling coefficient of the couplers used for their configurations. Another major point is that in CCORR configuration the loop power is nearly double of the value in the DCORR case.
The analytical results given in this article can be useful for selection of ORR configuration for particular applications, such as high power generation and investigation of nonlinear phenomena.

\section{REFERENCES}

[1] L. F. Stokes, M. Chodorow, and H. J. Shaw, "All-single-mode fiber resonator,” Opt. Lett., vol. 7, pp. 288-290, 1982.

[2] G. Soundra. Pandian and Faramarz E. Seraji, "Optical pulse response of a fibre ring resonator, " IEE Proc.-J, vol. 138 (3), pp. 235-239, 1991.

[3] M. Sumetsky, Y. Dulashko, and A. Hale, "Fabrication and study of bent and coiled free silica nanowires: Self-coupling microloop optical interferometer," Opt. Exp., vol. 12, pp. 3521-3531, 2004.

[4] M. Sumetsky, "Optical fiber microcoil resonator," Opt. Exp., vol. 12, pp. 2303-2316, 2004.

[5] Huilian Ma, Shijin Wang, and Zhonghe Jin, "Silica waveguide ring resonators with multi-turn structure," Opt. Commun., vol. 281, pp. 2509-2512, 2008.

[6] N. Talebi and M. Shahabadi, "Analysis of a lossy microring using the generalized multipole technique," Progress in Electromagnetics Research, PIER, vol. 66, pp. 287-299, 2006.

[7] G. Soundra Pandian and Faramarz E. Seraji; "Analysis of a fibre-optic ring resonator with polarization effects: Application to polarization," J. Mod. Opt., vol. 39 (5), pp. 991-1001, 1992.

[8] R. W. Boyd, J.H. Heebner, "Sensitive disk resonator photonic biosensor," Appl. Opt. vol. 40, pp. 5742-5747, 2001.

[9] Sai T. Chu, B. E. Little, Wugen Pan, Taro Kaneko, Shinay Sato, and Yasuo Kokubun, "An eight-channel add-drop filter using vertically coupled microring resonators over a cross grid," IEEE Photon. Technol. Lett., vol. 11 (6), pp. 691-693, 1999.

[10] D. G. Rabus, M. Hamacher, and H. Heidrich, "Active and passive microring resonator filter applications on GaInAsP/InP," Proc., 13th Int. Conf. on Indium Phosphide and Related Materials, IPRM, 14-18 May 2001, Nara, Japan, Pap. ThA1-3, pp. 477-480, 2001.

[11] Fufei Pang, Feng Liu, Xiuyou Han, Haiwen Cai, Ronghui Qu, Zujie Fang, "Analysis of a second-order polarization-independent filter made of a single ring resonator and a Sagnac interferometer," Opt. Commun., vol. 260, pp. 567-570, 2006.

[12] Sanjoy Mandal, Kamal Dasgupta, T.K. Basak, S.K. Ghosh, "A generalized approach for modeling and analysis of ring-resonator performance as optical filter," Opt. Commun., vol. 264, pp. 97-104, 2006.

[13] P.P. Yupapin, P. Saeung, C. Li, "Characteristics of complementary ring-resonator add/drop filters modeling by using graphical approach," Opt. Commun., vol. 272, pp. 81-86, 2007.

[14] P. Saeung, P.P. Yupapin, "Generalized analysis of multiple ring resonator filters: Modeling by using graphical approach," Optik, vol. 119 (10), pp. 465-472, 2008. 
[15] S. Dilwali and G. S. Pandian, "Pulse response of a fiber dispersion equalizing scheme based on an optical resonator," IEEE Photon. Technol. Lett., vol. 4 (8), pp. 942-944, 1992.

[16] Hao Shen, Jian-Ping Chen, Xin-Wan Li, Yi-Ping Wang, "Group delay and dispersion analysis of compound high order microring resonator all-pass filter," Opt. Commun., vol. 262, pp. 200-205, 2006

[17] Y. Dumeige, C. Arnaud, and P. Féron, "Combining FDTD with coupled mode theories for bistability in micro-ring resonators," Opt. Commun., vol. 250, pp. 376-383, 2005. (Erratum: Opt. Commun., vol. 272 p. 279, 2007).

[18] P. P. Yupapin, "Coupler-loss and coupling- coefficient- dependent bistability and instability in a fiber ring resonator," Optik, vol. 119 (10), pp. 492-494, 2008

[19] Otto Schwelb, "Crosstalk and bandwidth of lossy microring add/drop multiplexers," Opt. Commun., vol. 265, pp. 175-179, 2006.

[20] Junqing Li, Li Li, Jiaqun Zhao, and Chunfei Li, "Ultrafast, low power, and highly stable all-optical switch in MZI with two-arm-sharing nonlinear ring resonator," Opt. Commun., vol. 256, pp. 319-325, 2005.

[21] Rostami, "Low threshold and tunable all-optical switch using two-photon absorption in array of nonlinear ring resonators coupled to MZI," Microelectron. J., vol. 37, pp. 976-981, 2006.

[22] P. P. Yupapin, P. Chunpang, “An experimental investigation of the optical switching characteristics using an optical Sagnac interferometer incorporating one and two ring resonators," Opt. \& Laser Technol., vol. 40, pp. 273-277, 2008.

[23] J.L.S. Lima, K.D.A. Sabóia, J.C. Sales, J.W.M. Menezes, W.B. de Fraga, G.F. Guimarães, A.S.B. Sombra, "Optical short pulse switching characteristics of ring resonators," Opt. Fiber Technol., vol. 14 pp. 79-83, 2008.

[24] S. L. McCall, A. F. J. Levi, R. E. Slusher, S. J. Pearton, R. A. Logan, "Whispering-gallery mode microdisk lasers," Appl. Phys. Lett., vol. 60 pp. 289-291, 1992

[25] D. Garus, R. Hereth, F. Schliep, "Design considerations for Brillouin-Ringlaser gyroscopes," in: Conf. Proc. Symp. on Gyro Tech. (DGON). Stuttgart, p. 2.0, 1994.

[26] K. Kalli, D.A. Jackson, "Ring resonator optical spectrum analyzer with $20-\mathrm{kHz}$ resolution," Opt. Lett., vol. 17 (15), pp. 1090-1092, 1992.

[27] B.E. Little, S.T. Chu, J.V. Hryniewicz, P.P. Absil, "Filter synthesis for periodically coupled microring resonators," Opt. Lett., vol. 25 (5), pp. 344-346, 2000.

[28] T. Kominato, Y. Ohmori, N. Takato, H. Okazaki, M. Yasu, "Ring resonators composed of $\mathrm{GeO} 2$-doped silica wave- guides,” IEEE J. Lightwave Technol., vol. 12, pp. 1781-1788, 1992.

[29] R. Adar, Y. Shani, C.H. Henry, R.C. Kistler, G.E. Blonder, N.A. Olsson, "Measurement of very low-loss silica on silicon waveguides with a ring resonator," Appl. Phys. Lett., vol. 58 (5), pp. 444-445, 1991.

[30] K. Oda, S. Suzuki, H. Takahashi, H. Toba, "An optical FDM distribution experiment using a high finesse waveguide-type double ring resonator," IEEE Photon. Technol. Lett., vol. 6 (8), pp. 1031-1034, 1994.

[31] Faramarz E. Seraji, "New methods for rotation sensing by using a two-coupler fiber-optic ring resonator," Jap. J. Appl. Phys., Part 1, vol. 32 (4), pp. 1661-1667, 1993.

[32] Faramarz E. Seraji and G. S. Pandian, "Dynamic response of a fiber optic ring resonator with sinusoidal phase modulation of the loop," J. Mod. Opt., vol. 38, pp. 671-676, 1991.

[33] G. S. Pandian and Faramarz E. Seraji, "Dynamic analysis of a fibre-optic ring resonator excited by a sinewave-modulated laser diode," Jap. J. Appl. Phys., Part 1, vol. 29 (10), pp. 1967-1973, 1990.

[34] Diqing Ying, Huilian Ma, Zhonghe Jin, "Dynamic resonance characteristic analysis of fiber ring resonator," Opt. Fiber Technol., vol. 15, Issu. 1, pp. 15-20, 2009.

[35] Faramarz E. Seraji, "Steady-state performance analysis of fiber-optic ring resonator," Prog. Quant. Electron.(Elsevier), vol. 33, pp. 1-16, 2009.

[36] Faramarz E. Seraji, "Dynamic response of a fiber-optic ring resonator: Analysis with influences of light-source parameters,” Prog. Quant. Electron. (Elsevier), vol. 33, Issu. 2-4, pp. $110-125,2009$

[37] K. Saleh, P.H. Merrer, O. Liopis, and G. Cibiel, "Optical scattering noise in high $Q$ fiber ring resonators and its effect on optoelectronic oscillator phase noise", Opt. Lett., vol. 37 (4), pp. 518-520, 2012.

[38] Bo Chen, John Bracken, Frances Verleyen, Yong Wang, Chang-qing $\mathrm{Xu}$, "Analysis of all-optical intra-cavity wavelength conversions in fiber ring resonators", Opt. Commun., vol. 247, Iss. 1-3, pp 57-64, 2005.

[39] J. A. Bracken and C. Q. Xu, "All-Optical Wavelength Conversions Based on $\mathrm{MgO}$-Doped LiNbO3 QPM Waveguides Using an EDFA as a Pump Source" IEEE Photon. Technol. Lett., vol. 15, no. 7, pp. 954-956, 2003.

[40] T. Mizumoto and Y. Naito, "Dependence of the output phase on the asymmetry of 3-dB directional couplers" J. Lightwave Technol., vol. 8 (10), pp. 1571-1576, 1990. 